\title{
AVALIAÇÃO DA QUALIDADE DE VIDA DE APOSENTADOS COM A UTILIZAÇÃO DO QUESTIONÁRIO SF-36
}

\author{
Fausto Aloísio Pedrosa Pimenta, fabrícia fonseca Simil, Henrique Oswaldo da Gama Tôrres, Carlos faria Santos Amaral, Camila Farnese Rezende, \\ Thaissa Oliveira Coelho, Nilton Alves de Rezende*
}

Trabalho realizado na Faculdade de Medicina da Universidade Federal de Minas Gerais, Belo Horizonte, MG

\author{
*Correspondência \\ Faculdade de Medicina da \\ UFMG \\ Avenida Professor Alfredo \\ Balena, 190 \\ Santa Efigênia - Belo \\ Horizonte - Minas Gerais \\ CEP 30130-100 \\ Tel.: (31) 3547-2120 \\ Fax: (31) 3226-7738 \\ E-mail: \\ narezende@terra.com.br
}

\begin{abstract}
RESUMO
OBjetivo. O aumento da população idosa no Brasil justifica a necessidade de avaliar os aspectos que podem interferir na qualidade de vida de aposentados.

Métodos. A versão brasileira do questionário SF-36 foi aplicada em 87 aposentados para avaliação da qualidade de vida. Os resultados obtidos foram associados às características demográícas, socioeconômicas, condições de saúde e estilo de vida e foram estudados por meio de análise uni e multivariada.

Resultados. A idade média foi de 57,3 anos (desvio-padrão de 8,9 anos) e tempo médio de aposentadoria foi de 7,I anos. A aposentadoria foi por invalidez em $55,2 \%$ da amostra e $23,4 \%$ dos aposentados trabalhavam no momento da pesquisa. Os fumantes somaram $11,5 \%$ e 5,7\% eram dependentes de álcool. A depressão e hipertensão arterial sistêmica foram as doenças mais prevalentes, e 56,3\% dos aposentados praticavam algum tipo de atividade física regularmente. Após a análise multivariada, evidenciou-se melhor qualidade de vida apenas nos aposentados que praticavam atividade física regular ou que tinham alguma atividade de trabalho no momento da pesquisa.

Conclusão. $O$ questionário SF-36 foi um instrumento adequado, de aplicação relativamente rápida e de fácil uso para avaliação da qualidade de vida em aposentados. A qualidade de vida na amostra estudada foi associada ao estilo de vida dos pacientes e aponta para a necessidade de ações que contribuam de forma positiva para melhorar o estilo de vida nesta nova fase da vida.
\end{abstract}

UnItermos: Aposentadoria. Qualidade de vida. Idoso. Questionário SF-36.

\section{INTRODUÇÃO}

Vários estudos no Brasil e no mundo discutem o conceito de qualidade de vida - termo muitas vezes confundido com estilo de vida, condições de vida e situações de vida - e os instrumentos mais adequados para sua avaliação. Apesar das intensas discussões sobre o assunto, a definição de qualidade de vida ainda não é uniforme. Para melhor compreensão do conceito "qualidade de vida", deve-se diferenciá-lo do estado de saúde por meio de três dimensões principais: saúde mental, função física e função social. Para o estado de saúde, o fator mais importante é a função física. Para a qualidade de vida tornase importante também a saúde mental e o bem-estar psicológico e social. Dessa forma, instrumentos diferentes devem ser utilizados para a avaliação da qualidade de vida e do estado de saúde ${ }^{1,2}$.

A qualidade de vida pode estar diretamente associada à ausência de enfermidades, em especial à ausência de sintomas ou disfunções. Alguns autores, contudo, consideram este conceito reducionista, uma vez que aspectos não relacionados ao estado de saúde são considerados na avaliaçãa da qualidade de vida ${ }^{3}$.

A noção de qualidade de vida transita, portanto, em um campo semântico polissêmico: de um lado, encontra-se relacionada ao modo de vida, suas condições e estilos; de outro, inclui idéias sobre o desenvolvimento sustentável e sobre os direitos humanos e sociais. Estas noções se unem em uma resultante social de construção coletiva dos padrões de conforto e tolerância que determinada sociedade estabelece como referência?.

Tendências atuais enfatizam a subjetividade e o caráter multidimensional da qualidade de vida, resultado de políticas públicas e do desenvolvimento da sociedade em que os determinantes socioambientais se manifestam'. Dessa forma, a qualidade de vida passou a ser um indicador da eficácia e do impacto de determinados tratamentos, da comparação entre procedimentos para o controle de problemas de saúde, do impacto físico e psicossocial das enfermidades e da produção de conhecimentos decorrentes dos esforços de integração e intercâmbio entre profissionais e pesquisadores do tema².

Carr et al. (200 I $)^{4}$ enfatizam a necessidade de um foco individual na avaliação da qualidade de vida, chamando atenção para o "paradoxo da incapacidade", ou seja, pacientes com disfunções ou problemas de saúde importantes não apresentam, necessariamente, baixos escores nos questionários de avaliação da qualidade de vida.

As metodologias de avaliação da qualidade de vida baseiam-se em trabalhos tanto qualitativos como quantitativos. Os primeiros utilizam técnicas de biografias ou histórias de vida para evitar 
respostas estereotipadas. Os estudos quantitativos tendem para a construção e validação de instrumentos multidimensionais. Em ambos os casos são comumente utilizados questionários autoaplicáveis ou entrevistas com o próprio sujeito, e apresentam resultados comparáveis ${ }^{5}$. A tendência atual é a adaptação desses instrumentos para determinada enfermidade, como ocorre, por exemplo, com o MOS - HIV, para pacientes portadores da síndrome da imunodeficiência adquirida ${ }^{5}$.

Os principais indicadores utilizados para medir a qualidade de vida são bioestatísticos, psicométricos e econômicos, que não levam em conta o contexto cultural e social, a história de vida e o percurso dos indivíduos avaliados ${ }^{3}$. Entretanto, os instrumentos de avaliação da qualidade de vida não devem utilizar medidas focalizadas apenas nos sintomas, mas devem incluir itens qualitativos como os utilizados em pesquisas sociológicas ${ }^{1,6}$. Embora a qualidade de vida venha sendo estudada em diversos grupos sociais, ela é pouco conhecida em aposentados, principalmente no Brasil.

O Short Form-36 (SF-36) é um instrumento de medida de qualidade de vida desenvolvido no final dos anos 80 nos EUA. Foi aplicado em diversas situações com boa sensibilidade, eliminando-se o problema de distribuição excessiva das pontas de escala como excelente e muito ruim $^{7,8}$. Este instrumento foi traduzido e validado no Brasil para avaliar a qualidade de vida em pacientes com artrite reumatóide e mostrou-se adequado às condições socioeconômicas e culturais da população brasileira?. Por este motivo, o SF-36 foi o instrumento escolhido para estimar a qualidade de vida de aposentados avaliados neste estudo.

\section{Métodos}

Trata-se de um estudo epidemiológico observacional do tipo transversal. A população estudada foi constituída pelo universo de aposentados de ambos os sexos vinculados a um plano de saúde de autogestão de uma empresa estatal de Minas Gerais, residentes em Belo Horizonte. No momento da pesquisa, essa operadora de plano de saúde contava com 187 aposentados associados e a amostra foi calculada em 87 indivíduos. Estes foram escolhidos por meio de tabela de números aleatórios. As variáveis independentes selecionadas foram equivalentes às que têm sido tradicionalmente utilizadas em pesquisas sobre fatores relacionados à qualidade de vida pelo SF-36. O SF-36 é composto por II questões e 36 itens que englobam oito componentes (domínios ou dimensões), representados por capacidade funcional (dez itens), aspectos físicos (quatro itens), dor (dois itens), estado geral da saúde (cinco itens), vitalidade (quatro itens), aspectos sociais (dois itens), aspectos emocionais (três itens), saúde mental (cinco itens) e uma questão comparativa sobre a percepção atual da saúde e há um ano. 0 indivíduo recebe um escore em cada domínio, que varia de 0 a 100 , sendo 0 o pior escore e 100 o melhor ${ }^{10}$.

\section{Aspectos demográficos, antropométricos e socioeconômicos}

A população foi avaliada quanto ao sexo, faixa etária (30 a 39 anos; 40 a 49 anos; 50 a 59 anos; 60 a 69 anos; acima de 70 anos), estado conjugal (solteiro, casado ou em união consensual, divorciado ou separado e viúvo), arranjo familiar (morar sozinho ou acompanhado) e nível socioeconômico investigado por meio da escolaridade e renda. A escolaridade foi avaliada de acordo com o número de anos estudados e a renda familiar mensal foi calculada pela soma das rendas da aposentadoria e/ou pensão, acrescida da ajuda de familiares e rendimentos de outras fontes, sendo calculado o número de salários mínimos com base no valor de janeiro de 2006 ( $R \$ 280,00)$.

\section{Dados da aposentadoria}

A aposentadoria foi classificada quanto ao seu tipo, por invalidez e por tempo de serviço. A aposentadoria por invalidez foi concedida ao funcionário incapacitado para o trabalho por motivo de saúde ou por acidente de trabalho. A aposentadoria por tempo de serviço foi considerada ao completar-se 30 anos de serviço, para homens, e 25 para as mulheres. Os dados sobre a aposentadoria foram obtidos nos registros cadastrais do plano de saúde.

A aposentadoria ocorrida por invalidez foi subdividida em aposentadorias ocasionadas por doenças osteomusculares relacionadas ao trabalho (DORT), por doenças profissionais ou por doenças clínicas. O tempo de aposentadoria foi calculado em anos, a partir da sua data, utilizando-se como ponto de corte a mediana "sete anos". A ocupação após a aposentadoria foi classificada em relação ao tipo de atividade e à existência ou não de remuneração que não a auferida pela aposentadoria.

\section{Dados sobre a situação de saúde e estilo de vida}

A atividade física foi caracterizada como regular se era realizada com freqüência superior a cinco vezes por semana e com duração igual ou maior que 30 minutos por dia". As doenças crônicas analisadas foram depressão, artrite/reumatismo, câncer, doença neurológica crônica (exceto acidente vascular encefálico - AVE), hipertensão arterial sistêmica (HAS), diabetes melito, asma/bronquite/enfisema, AVE, deficiência visual incapacitante ou deficiência auditiva incapacitante. A prevalência do tabagismo foi caracterizada em: nunca fumou (menos de 100 cigarros durante a vida); já fumou, (pelo menos 100 cigarros durante a vida) e fumante (mais de 100 cigarros durante a vida e consumo atual), de acordo com National Center for Health Statistics, 1994, já utilizado no Brasil por Peixoto (2005) ${ }^{12}$. O consumo de álcool foi avaliado com base nos critérios do questionário "CAGE" 13.

\section{Coleta de dados}

A coleta de dados foi realizada no período de outubro de 2005 a abril de 2006, pelo pesquisador principal e por duas estudantes de Medicina, em entrevistas agendadas com os aposentados em sua residência. Para verificar a concordância na coleta de dados entre os pesquisadores, foi calculada a estatística Kappa entre os mesmos, que foi de 0,69 , considerada uma boa concordância ${ }^{14}$. Os dados obtidos foram digitados (e conferidos para evidenciar erros) no programa Eplnfo 6.04 (CDC, Atlanta, Domínio Público).

\section{Metodologia estatística}

O cálculo dos escores do SF-36 foi feito de acordo com os seguintes passos:

I. Cálculo de cada um dos domínios (capacidade funcional, aspectos físicos, dor, estado geral da saúde, vitalidade, aspectos 
sociais, aspectos emocionais e saúde mental) e soma dos pontos obtidos em cada item relativo ao domínio correspondente, para cada aposentado.

2. Uso dos valores mínimos e máximos possíveis em cada item para calcular o valor transformado, com o emprego da fórmula:

$$
Y_{i j}=\frac{X_{i j}-\min j}{\text { Máxj - minj }} \times 100
$$

Em que: $\mathrm{i}=1,2,3, \ldots .87$ (índice do aposentado); $j=$ Capacidade funcional, aspectos físicos... (cada um dos domínios); Yij = valor transformado do aposentado i, domínio j; Xij = valor do domínio j, do aposentado i; Mín $\mathrm{j}$ = valor mínimo possível para o domínio j; Máx j = valor máximo possível para o domínio j.

A comparação com outras variáveis categóricas foi feita por meio de tabelas de contingência. Utilizou-se o teste Qui-quadrado com correção de Yates para comparação de proporções. Quando uma das freqüências esperadas foi inferior a cinco, utilizou-se o teste de Fisher. O nível de significância estatística estabelecida foi $p<0,05$, com importante tendência de significância estatística para valores entre 0,10 e 0,05 . Os escores de pontuação dos domínios do SF-36 foram comparados com variáveis categóricas por meio do teste Kruskal-Wallis. A análise multivariada foi feita por meio da regressão linear pelo programa MINITAB, versão |I.2. Inicialmente foram incluídas no modelo as variáveis com valor de $p<0,25$ na análise univariada. Em seguida, as variáveis com menor nível de significância foram retiradas do modelo, até que restassem apenas variáveis com significância estatística com valor de $p<0,05$. Foram desenvolvidos oito modelos de regressão linear, sendo um para cada domínio do SF-36.

\section{Comitê de ética}

Este estudo foi aprovado pelo Comitê de Ética em Pesquisa da Universidade Federal de Minas Gerais (COEP/UFMG), parecer ETIC 179/05.

\section{Resultados}

Foram entrevistados 87 aposentados sorteados por meio de tabela de números aleatórios. Após o sorteio, 13 aposentados foram substituídos, também de forma aleatória, em decorrência de alcoolismo com incapacidade de preencher o formulário (I), doença de Alzheimer (I), não concordância em participar da pesquisa (8) e erros nos dados cadastrais (3). Não houve diferença estatística da idade, sexo e tipo de aposentadoria entre os aposentados entrevistados e os excluídos.

A descrição da amostra quanto aos dados demográficos e socioeconômicos dos 87 aposentados entrevistados encontra-se na Tabela I.

Sessenta e quatro por cento dos aposentados foram classificados como fumantes ( I I,5\%) ou ex-fumantes (52,9\%), e 35,6\% como nãofumantes. De acordo com o questionário CAGE, 5,7\% dos entrevistados foram considerados dependentes de álcool. As doenças mais comuns apresentadas pelos aposentados foram depressão $(24,1 \%)$ e hipertensão arterial sistêmica (39\%), seguida por diabetes melito (10\%) e doenças articulares (9\%) (Tabela 2).

\begin{tabular}{|c|c|c|}
\hline \multicolumn{3}{|c|}{$\begin{array}{l}\text { Tabela I - Características demográficas e socioeconômicas de uma } \\
\text { população de aposentados de Belo Horizonte, MG }(\mathrm{n}=87)\end{array}$} \\
\hline Característica & $n$ & $\%$ \\
\hline $\begin{array}{l}\text { Sexo } \\
\text { Masculino } \\
\text { Feminino }\end{array}$ & $\begin{array}{l}58 \\
29\end{array}$ & $\begin{array}{l}66,7 \% \\
33,3 \%\end{array}$ \\
\hline $\begin{array}{l}\text { Idade (anos) } \\
\text { Média } \\
30-49 \\
50-59 \\
\geq 60\end{array}$ & $\begin{array}{l}57,3 \text { anos } \\
20 \\
37 \\
32\end{array}$ & $\begin{array}{r}22,4 \% \\
41,6 \% \\
36 \%\end{array}$ \\
\hline $\begin{array}{l}\text { Estado conjugal } \\
\text { Casado ou em união consensual } \\
\text { Outros }\end{array}$ & $\begin{array}{l}60 \\
27\end{array}$ & $\begin{array}{l}69 \% \\
31 \%\end{array}$ \\
\hline $\begin{array}{l}\text { Mora sozinho } \\
\text { Sim } \\
\text { Não }\end{array}$ & $\begin{array}{c}9 \\
78\end{array}$ & $\begin{array}{l}10,3 \% \\
89,7 \%\end{array}$ \\
\hline $\begin{array}{l}\text { Escolaridade (anos estudados) } \\
\text { Média } \\
\leq 8 \\
9 \text { a } 11 \\
\geq 12\end{array}$ & $\begin{array}{l}10,7 \text { anos } \\
26 \\
27 \\
34\end{array}$ & $\begin{array}{r}29,9 \% \\
31 \% \\
39,1 \%\end{array}$ \\
\hline $\begin{array}{l}\text { Renda familiar (salários mínimo } \\
\leq 3 \\
>3 \\
\text { Seminformação }\end{array}$ & $\begin{array}{l}30 \\
55 \\
2\end{array}$ & $\begin{array}{l}63,2 \% \\
34,5 \% \\
2,3 \%\end{array}$ \\
\hline
\end{tabular}

Os piores escores de qualidade de vida foram observados quanto aos domínios "aspectos físicos", "dor" e "estado geral de saúde" (Tabela 3).

O sexo masculino apresentou um escore maior de pontuação, exceto para o estado geral de saúde e aspectos emocionais. Quanto à idade, houve diferença para a capacidade funcional, dor, vitalidade, aspectos sociais e saúde mental. Maiores pontuações foram observadas nos aposentados de idade mais avançada, ou seja, os mais idosos apresentaram melhor qualidade de vida. Aposentados casados apresentaram maior pontuaçãa nos domínios "capacidade funcional", "aspectos físicos, "estado geral de saúde", "vitalidade", "aspectos sociais" e "saúde mental". Não houve diferença da qualidade de vida nos aposentados que moravam sozinhos, ou em relação a características como escolaridade e renda familiar. A qualidade de vida dos aposentados por invalidez mostrou-se inferior, com menor pontuação para todos os domínios do que aquela por tempo de serviço. A qualidade de vida dos aposentados com ocupação após a aposentadoria apresentou melhor pontuação em todos os domínios do SF-36. O tempo de aposentadoria não esteve relacionado à qualidade de vida. A prática de atividade física de forma regular esteve associada com melhor qualidade de vida nos domínios "capacidade funcional", "estado geral de saúde", "vitalidade", "aspectos sociais" e "saúde mental". Aposentados com depressão apresentaram pior qualidade de vida, com menor pontuação em todos os domínios. A artrite associou-se a uma menor pontuação nos domínios "capacidade funcional", "aspectos emocionais e físicos". A doença neurológica crônica ou HAS associou-se a um 


\begin{tabular}{|c|c|c|}
\hline Característica & $\mathrm{n}$ & $\%$ \\
\hline $\begin{array}{l}\text { Aposentadoria } \\
\text { Invalidez } \\
\text { Tempo de serviço }\end{array}$ & $\begin{array}{l}48 \\
39\end{array}$ & $\begin{array}{l}55,2 \% \\
44,8 \%\end{array}$ \\
\hline $\begin{array}{l}\text { Causas da aposentadol } \\
\text { DORT } \\
\text { Outras }\end{array}$ & $\begin{array}{l}29 \\
19\end{array}$ & $\begin{array}{l}60,4 \% \\
39,6 \%\end{array}$ \\
\hline $\begin{array}{l}\text { Tempo de aposentadoI } \\
\qquad \begin{array}{l}\mid-6 \\
\geq 7\end{array}\end{array}$ & $\begin{array}{l}45 \\
42\end{array}$ & $\begin{array}{l}51,7 \% \\
48,3 \%\end{array}$ \\
\hline $\begin{array}{l}\text { Atividade de trabalho } \\
\text { Sim } \\
\text { Não }\end{array}$ & $\begin{array}{l}23 \\
64\end{array}$ & $\begin{array}{l}26,4 \% \\
73,6 \%\end{array}$ \\
\hline $\begin{array}{l}\text { Atividade de trabalho } \\
\text { Remunerada } \\
\text { Não }\end{array}$ & $\begin{array}{c}15 \\
8\end{array}$ & $\begin{array}{l}65,2 \% \\
34,8 \%\end{array}$ \\
\hline $\begin{array}{l}\text { Atividade física habitua } \\
\text { Sim } \\
\text { Não }\end{array}$ & $\begin{array}{l}49 \\
38\end{array}$ & $\begin{array}{l}56,3 \% \\
43,7 \%\end{array}$ \\
\hline $\begin{array}{l}\text { Tipo de atividade física } \\
\text { Caminhada } \\
\text { Outros }\end{array}$ & $\begin{array}{l}41 \\
8\end{array}$ & $\begin{array}{l}84,5 \% \\
15,5 \%\end{array}$ \\
\hline $\begin{array}{l}\text { Duração da atividade } \mathrm{f} \\
\quad<30 \\
\geq 30\end{array}$ & $\begin{array}{c}4 \\
45\end{array}$ & $\begin{array}{l}8,2 \% \\
91,8 \%\end{array}$ \\
\hline $\begin{array}{l}\text { Tabagismo } \\
\text { Nuncafumou } \\
\text { Jáfumou } \\
\text { Fumante }\end{array}$ & $\begin{array}{l}31 \\
46 \\
10\end{array}$ & $\begin{array}{l}35,6 \% \\
52,9 \% \\
11,5 \%\end{array}$ \\
\hline $\begin{array}{l}\text { Uso de álcool* } \\
\text { Dependente de álcool }\end{array}$ & 5 & $5,7 \%$ \\
\hline
\end{tabular}

menor escore para "capacidade funcional", enquanto os aposentados com diabetes melito apresentaram também menor pontuação no componente "estado geral de saúde".

O Quadro I apresenta o resumo dos fatores associados à qualidade de vida após a análise multivariada.

\section{Discussão}

Aposentados do sexo masculino apresentaram, com exceção dos domínios "estado geral de saúde" e "aspectos emocionais", maiores escores nos outros domínios avaliados pelo SF-36, com conseqüente melhor avaliação da qualidade de vida. Ressalta-se que as mulheres apresentaram tendência a considerar sua saúde pior quando comparada aos homens da mesma faixa etária, aspectos que também foram relatados em outros estudos ${ }^{15,16}$.

A idade apresentou diferença para os domínios "capacidade funcional", "dor", "vitalidade", "aspectos sociais" e "saúde mental", sempre com pontuações maiores para os aposentados de idade mais avançada, o que implica melhor qualidade de vida. Isso pode ser explicado com

\begin{tabular}{|c|c|c|c|c|c|}
\hline \multicolumn{6}{|c|}{$\begin{array}{c}\text { Tabela } 3 \text { - Valores dos domínios avaliados pelo SF-36 em uma } \\
\text { população de aposentados de Belo Horizonte, MG }(n=87)\end{array}$} \\
\hline Variável & Média & $\begin{array}{l}\text { Desvio- } \\
\text { padrão }\end{array}$ & Mínimo & Mediana & Máximo \\
\hline Capacidade funcional & 61,1 & 26,2 & 0,0 & 65,0 & 100,0 \\
\hline Aspectos físicos & 49,2 & 40,9 & 0,0 & 50,0 & 100,0 \\
\hline Dor & 54,8 & 22,9 & 12,0 & 52,0 & 100,0 \\
\hline Estado geral de saúde & 59,0 & 20,0 & 10,0 & 57,0 & 92,0 \\
\hline Vitalidade & 61,0 & 21,7 & 15,0 & 60,0 & 100,0 \\
\hline Aspectos sociais & 70,0 & 21,4 & 12,5 & 75,0 & 100,0 \\
\hline Aspectos emocionais & 53,4 & 40,8 & 0,0 & 66,7 & 100,0 \\
\hline Saúdemental & 68,4 & 21,8 & 12,0 & 72,0 & 100,0 \\
\hline
\end{tabular}

\section{Quadro I - Fatores associados à qualidade de vida com base na análise multivariada em uma população de aposentados de Belo Horizonte, MG $(n=87)$}

\begin{tabular}{|c|c|}
\hline $\begin{array}{l}\text { Fatores que influenciam } \\
\text { positivamente a qualidade de vida }\end{array}$ & $\begin{array}{l}\text { Fatores que influenciam } \\
\text { negativamente a qualidade de vida }\end{array}$ \\
\hline $\begin{array}{l}\text { Aposentadoria por tempo de serviço }{ }^{a} \\
\text { Ocupação após a aposentadoria }{ }^{b} \\
\text { Atividade física regular }^{\text {a }} \\
\text { Sexo masculino }{ }^{a} \\
\text { Idade avançada }\end{array}$ & $\begin{array}{l}\text { Aposentadoria por invalidez }^{a} \\
\text { Ausência de ocupaçãa após a aposentadoria }{ }^{b} \\
\text { Depressão }^{a} \\
\text { Aposentar-se jovem }^{\text {a }}\end{array}$ \\
\hline
\end{tabular}

base no motivo da aposentadoria dos mais jovens que, em sua maioria, foi por invalidez (55,2\%). Deve-se salientar a possibilidade do viés de sobrevivência, tendo em vista que aposentados com piores condições de saúde tenderiam a morrer mais cedo. Alguns autores, utilizando outros instrumentos de avaliação, também encontraram resultados semelhantes ${ }^{17,18}$, devendo ser salientado que a relação "qualidade de vida" e "envelhecimento" envolve variáveis como atividade física, renda, vida social e relações familiares ${ }^{19}$.

Os aposentados casados ou em união consensual apresentaram maior pontuação nos domínios "capacidade funcional", "aspectos físicos", "estado geral de saúde", "vitalidade", "aspectos sociais" e "saúde mental". Assim, apresentaram melhor qualidade de vida quando comparados aos separados ou viúvos, o que está de acordo com o descrito na literatura ${ }^{20}$

Os entrevistados que exerciam atividade de trabalho após a aposentadoria apresentaram maior pontuação em todos os domínios avaliados pelo SF-36. Embora autores como Bossé et al. (1987)21 não tenham encontrado diferenças quanto aos sintomas psicológicos nos aposentados com ocupação ou não após a aposentadoria. Gonzáles et al. (1995)22 relataram piora na saúde mental em aposentados inativos. Reitzes et al. (1996)23 também observaram melhora da saúde mental e dos aspectos emocionais em aposentados que mantinham algum tipo de ocupação após a aposentadoria. Herzog et al. (|99|) ressaltaram que a forma de exercício da atividade de trabalho após a aposentadoria pode influir na qualidade de vida, destacando que o trabalho estressante contribuiu para uma piora da saúde mental em aposentados ${ }^{24}$.

A prática regular de atividade física apresentou diferença com significância estatística para uma maior pontuação nos domínios 
"capacidade funcional", "estado geral de saúde", "vitalidade", "aspectos sociais" e "saúde mental", estando de acordo com outros estudos que associaram a atividade física a uma melhora da qualidade de vida ${ }^{25,26}$.

Aposentados que relataram doença neurológica crônica ou HAS apresentaram menor escore para o domínio "capacidade funcional". Os que relataram diabetes melito apresentaram tendência importante de menor pontuação para o domínio "estado geral de saúde". Entre os aposentados que relataram deficiência visual incapacitante, observouse tendência importante de menor pontuação para os domínios "capacidade funcional", "aspectos físicos", "vitalidade" e "aspectos emocionais". Não foi observada diferença em nenhum domínio para os aposentados que relataram câncer ou asma/bronquite/enfisema. Esses dados estão, também, de acordo com o relatado na literatura ${ }^{27-30}$

Dos domínios avaliados, ressalta-se a manutenção da capacidade funcional, que por sua vez está diretamente associada à qualidade de vida, pois refere-se à capacidade de um indivíduo se manter na comunidade com independência, sendo que esse domínio apresenta associação satisfatória com a avaliação clínica ${ }^{3 !}$.

\section{ConClusão}

O questionário SF-36 mostrou-se um instrumento adequado para a avaliação da qualidade de vida na amostra de aposentados estudada.

Estudos transversais, como o realizado, impossibilitam a associação causal, pois apenas sugerem a inter-relação entre as condições de saúde auto-referidas, a qualidade de vida e a situação no mercado de trabalho, cuja temporalidade só poderá ser avaliada em outros tipos de estudos. Por esta razão, estudos longitudinais devem ser realizados com o objetivo de obter maiores explicações sobre a causalidade das relações entre aposentadoria por invalidez ou tempo de serviço com a qualidade de vida.

\section{Conflito de interesse: não há}

\section{SUMMARY}

\section{Retiree Quality of life assessment with SF-36 QUESTIONNAIRE}

OBIECTIVE. Increase of the elderly in the Brazilian population demands assessment of aspects that interfere with the retirees' quality of life.

METHODS. The Brazilian version ofSF-36 questionnaire was applied to 87 retirees at their homes. Data on quality of life was associated with demographic data, socioeconomic status, health conditions and life style. Statistical analysis of data was performed using single-variable and multivariate analysis.

RESULTS. The mean age was 57.3 years (standard deviation 8.9 years) and the mean retirement time was 7 . I years. Fifty-five percent were early retirements due to disability and $23.4 \%$ of those queried were still working when research was carried out. Smokers were $11.5 \%$ and $5.7 \%$ were diagnosed as alcohol dependent by CAGE criteria. Depression and arterial systemic hypertension were the most prevalent conditions and $56.3 \%$ of the retirees practiced regular physical activity. Multivariate analysis disclosed that regular physical activity and post-retirement occupation were the only variables associated with improved quality of life.
CONCLUSION. The SF-36 questionnaire was a suitable instrument, relatively quick and easy to use. The quality of life was associated with life style, as indicated by the practice of physical activities and post-retirement occupation. Actions are needed to enable retirees to improve their life style after retirement. [Rev Assoc Med Bras 2008; 54(I): 55-60]

KEY wORDS: Retirement. Quality of life. Elderly. SF-36 questionnaire.

\section{REFERÊNCIAS}

I. Rocha AD, Okabe I, Martins MEA, Machado PHB, Mello TC. Qualidade de vida, ponto de partida ou resultado final? Ciênc. saúde coletiva 2000; 5:63-8I.

2. Seidl EMF, Zannon CMLC. Qualidade de vida e saúde: aspectos conceituais e metodológicos. Cad de Saude Publica 2004; 20:580.

3. Minayo MCS, Hartz ZMA, Buss PM. Qualidade de vida e saúde: um debate necessário. Ciênc. saúde coletiva 2000; 5:7-18.

4. Carr JA, Higginson IJ. Measuring quality of life: are quality of life measures patient centred? Br Med J 200 I; 322: I 357-1360.

5. Wu AW, Hays RD, Kelly S, Malitz F, Bozzette SA. Aplicattions of the medical outcomes study health-related quality of life measures in HIV/ AIDS. Qual Life Res 1997; 6:53 I-554.

6. Gill TM, Alvan ND, Feinstein MD. A critical appraisal of the quality of quality-of-life measurements. J Am Med Assoc 1994; 272:61 9-626.

7. Ware JE, Sherbourne CD. The MOS - 36 item Short Form Health Survey (SF - 36) conceptual framework and item selection. Med Care 1992; 30:473-483.

8. Viacava F. Informações em saúde: a importância dos inquéritos populacionais. Ciênc. saúde coletiva 2002; 7:607-621.

9. Ciconelli RM, Ferraz MB, Santos W, Meinão I, Quaresma MR. Tradução para a língua portuguesa e validação do questionário genérico de avaliação de qualidade de vida SF-36 (Brasil SF-36). Rev Bras Reumatol 1999; 39: $143-150$.

10. Ware JE, Kosinski M, Gandek B. SF-36 Health Survey: Manual \& Interpretation Guide. Lincoln, RI: QualityMetric Incorporated, 2003.

I I. Pate RR, Pratt M, Blair SN, Haskell WL, Macera CA, Bouchard C, et al. Physical activity and public health. A recommendation from the Centers for Disease Control and Prevention and the American College of Sports Medicine. JAMA 1995; 273:402-407.

12. Peixoto, S. V.; Firmo, J. O. A.; Lima-Costa, M. F. Factors associated to smoking habit among older adults (The Bambuí Health and Aging Study). Cad de Saude Publica, v.39, n.5, p.745-753, 2005.

13. Masur, J.; Monteiro, M. Validation of the CAGE alcoholism screening test in Brazilian Psychiatry Inpatient Hospital Setting. Journal Biology Research, v. 16, p.215-8, 1983.

14. Jekel, JF; Elmore, JG; Katz, DL. Epidemiologia, Bioestatistica e Medicina Preventiva. Porto Alegre: Artes Médicas, 1999. 328p. il.

15. Kubzansky LD, Berkman LF, Glass TA, Seeman TE. Is educational attainment associated with shared determinants of health in the elderly? Findings from the MacArthur studies of successful aging. Psychosom Med 1998; 60:578-585.

16. Mcdonough P, Walters V. Gender and health: reassessing patterns and explanations. Soc Sci Med 200 I ; 52:547-559.

17. Eden L, Ejlertsson G, Leden I. Health and health care utilization among early retirement pensioners with musculoskeletal disorders. Scand J Prim Health Care 1995; 12:211-216.

18. Elovainio M, Kivimäki M, Vahtera J, Ojanlatva A, Korkeila K, Suominen S, Helenius $H$, Koskenvuo M. Social support, early retirement, and a retirement preference: a study of 10,489 finnish adults. J Occup Environ Med 2003; 45:433-439.

19. Xavier FMF, Ferraz MPT, Marc N, Escosteguy NU, Moriguchi E. A definição dos idosos de qualidade de vida. Rev Bras Psiquiatr 2003; 25:31-39.

20. Berkman LF, Syme SL. Social networks, host resistance, and mortality: a nine-year follow-up study of Alameda County residents. Am J Epidemiol 1979; 109: 1 86-204. 
Pimenta fap et al.

21. Bossé R, Aldwin C, Levenson M, Ekerdt D. Mental health differences among retirees and workers: findings from the Normative Aging Study. Psychol Aging 1987; 2:383-389.

22. Gonzáles FAL, Mota-Velazco G, Salas-Ro-Mero MF. Depression en jubilados no activos. Estudio de 234 casos. Rev Med 1995; 33:5 I-55.

23. Reitzes DC, Mutran EJ, Fernandes ME. Does retirement hurt well-being? Factors influencing self-steem and depression among retirees and workers. Gerontologist 1996; 36:649-656.

24. Herzog AR, House JS, Morgan JN. Relation of Work and Retirement to Health and Well-Being in Older Age. Psychol Aging 1991; 6:202-211.

25. Caspersen CJ, Pereira MA, Curran KM. Changes in physical activity patterns in the United States, by sex and cross-sectional age. Med Sci Sports Exerc 2000; 32:1601-1609.

26. Valderrábano F. Quality of life benefits of early anaemia treatment. Nephrol Dial Transplant 2000; 15:23-28.

27. Ferraro KF, Farmer MM, Wybraniec JA. Health Trajectories: Longterm Dynamics Among Black and White Adults. J Health Soc Behav 1997; 38:38-54.
28. Hoeymans N, Feskens EJM, Kromhout D, Van Den Bos GAM. Ageing and the relationship between functional status and self-rated health in elderly men. Soc Sci Med 1997; 45: I 527- 1536.

29. Damián J, Ruigomez A, Pastor V, Martin-Moreno JM. Determinants of self assessed health among Spanish older people living at home. J Epidemiol Community Health 1999; 53:4I2-4I6.

30. Gama EV, Damián J, Del Molino JP, López MR, Pérez ML, Iglesias FJG. Association of individual activities of daily living with self-rated health in older people. Age Ageing 2000; 29:267-270.

31. Veras RP. Em busca de uma assistência adequada à saúde do idoso: revisão da literatura e aplicação de um instrumento de detecção precoce e de previsibilidade de agravos. Cad de Saude Publica 2003; 19:705-7I 5.

Artigo recebido: 26/06/07

Aceito para publicação: 31/10/07 